Apidologie, 1974, 5 (3), 201-223.

\title{
ANALYSE PAR RADIOACTIVATION DU CONTENU MINÉRAL D'UN MIEL. POSSIBILITÉ DE PRÉCISER SON ORIGINE GÉOGRAPHIQUE
}

\author{
Analyse des Mineralgehaltes eines Honigs \\ mittels Radioaktivierung \\ Möglichkeit einer genauen geographischen \\ Herkunftsbestimmung eines Honigs
}

\author{
G. LASCEVE et M. GONNET* \\ Département de Biologie, Service de Radioagronomie, CEN Cadarache, \\ $B P$ No 1, 13115 Saint-Paul-lez-Durance \\ * Station expérimentale d'Apiculture, \\ Centre de Recherches Agronomiques d'Avignon, I.N.R.A., \\ 84140 Montfavet
}

\author{
SUMMARY \\ RADIOACTIVATION ANALYSIS OF THE MINERAL CONTENT OF A HONEY. \\ POSSIBILITY OF PRECISING THE GEOGRAPHIC ORIGIN.
}

The aim of the present study consists in searching whether there is a relation between the mineral composition of a honey and its geographic origin. The trace analysis is carried out by activation analysis with thermic neutrons on a $15 \mathrm{~g}(0,53$ ounce) sample reduced to ashes under infra-red radiations. So about twenty elements can be recognized without resorting any chemical separation. The 15 samples analysed contain the same trace elements but in proportions which are different enough to make possible the identification of each origin. Moreover the ratios $\mathrm{Rb} / \mathrm{Fe}$ and $\mathrm{Rb} / \mathrm{Zn}$ allow to discriminate between light honeys and dark honeys and between Hungarian and French locusttree honeys. The use of a single element as indicator seems to be possible in a few special cases : the fir honey from the Loire area is the only one which contains silver.

This memoir forms a preliminary study which must be precised and deepened by the analysis of several samples from the same place but harvested at different times in order to determine the variation boundaries of the various elements.

\section{RÉSUMÉ}

L'objet du présent travail consiste à rechercher s'il existe des liens entre la composition minérale d'un miel et son origine géographique. L'analyse des traces est réalisée par analyse par activation aux neutrons thermiques sur un échantillon de 15 grammes réduit en cendres 
sous infra-rouge. Une vingtaine d'éléments peuvent être ainsi reconnus sans avoir recours à aucune séparation chimique. Les quinze échantillons analysés contiennent les mêmes traces mais dans des proportions suffisamment différentes pour qu'il soit possible d'identifier chaque origine. Les rapports $\mathrm{Rb} / \mathrm{Fe}$ et $\mathrm{Rb} / \mathrm{Zn}$ permettent en outre une distinction entre miels clairs et miels foncés et la différenciation entre les miels d'acacia hongrois et français. L'utilisation d'un seul élément comme indicateur ne semble possible que dans quelques cas particuliers : le miel de sapin de la Loire est seul à contenir de l'argent.

Ce mémoire constitue un travail préliminaire qui doit être précisé et approfondi par l'analyse de plusieurs échantillons provenant du même site mais récoltés à différentes époques afin de déterminer les limites de variations des différents éléments.

\section{INTRODUCTION}

La recherche de l'origine florale et de l'origine géographique des miels reste, malgré les progrès accomplis dans l'analyse pollinique (LouveAux J., 1968; Louveaux et al., 1970) et dans la chromatographie en phase gazeuse des sucres (Pourtaluer J., 1967) un domaine où l'interprétation des résultats n'est pas toujours sans difficultés. En général on ne se contente pas des résultats provenant d'une méthode unique; on cherche au contraire à obtenir plus de précision et plus de sûreté par des recoupements en utilisant des méthodes indépendantes reposant sur des principes différents. Ainsi la caractérisation d'un miel de callune pourra faire appel à des mesures physiques (viscosité), chimiques (dosage des protéines) et pollinique (recherche des pollens les plus significatifs).

Dans cet esprit l'identification et le dosage des constituants minéraux du miel paraissent susceptibles de contribuer à l'affinement des méthodes déjà en usage en apportant des éléments originaux et peut-être très caractéristiques. Des produits tels que l'opium (Pappas A. C., 1963) et la marijuana (BRYAN D. E., 1967) ont déjà fait l'objet de recherches de cette nature mais, sur le miel, peu d'études en vue de relier provenance et composition minérale ont été réalisées bien que de nombreuses analyses aient montré des différences de composition entre des échantillons de diverses origines. Vermeulen L. et Pelerents C. (1965) font état des corrélations entre date de récolte, région, couleur de nombreux miels belges et leur teneur en hydrates de carbone, en phosphore et en fer. Miskiewicz W. (1969) indique pour différents miels polonais, des variations de concentrations en calcium, phosphore, manganèse et fer selon leur origine. Les teneurs en sels minéraux de miels d'acacia hongrois et leur corrélation avec plante et sol sont étudiées par VARJU M. (1970).

Les sucres constituants essentiels du miel, rendent quelque peu difficile l'analyse des nombreux oligo-éléments présents. Cependant depuis le début du siècle le nombre de traces reconnues et dosées n'a cessé de grandir. VAN Dine D. L. et Thomson A. R. en 1908 signalent et dosent le calcium et le magnésium, puis Fenlmann C. (1912) le phosphore. Schuette H. A. ajoute en 1932, 1938 et 1939 les éléments suivants : $\mathrm{Fe}, \mathrm{Cu}, \mathrm{Mn}, \mathrm{Si}, \mathrm{S}, \mathrm{Cl}, \mathrm{Na}$ et $\mathrm{K}$. Mladenov S. 
(1968) et Petrov V. (1970), étudiant le miel pour sa valeur nutritive complètent cette liste avec 21 nouveaux éléments : $\mathrm{Al}, \mathrm{Be}, \mathrm{B}, \mathrm{Ba}, \mathrm{Ge}, \mathrm{Ga}, \mathrm{Au}, \mathrm{Sn}, \mathrm{Co}, \mathrm{Li}$, $\mathrm{Mo}, \mathrm{Pb}, \mathrm{Ag}, \mathrm{Sr}, \mathrm{Ti}, \mathrm{Cr}, \mathrm{Zn}, \mathrm{Zr}$, et Sb, et proposent d'utiliser le miel pour compenser certaines carences en oligo-éléments chez l'homme.

Devant cette abondance de traces minérales et avant d'entreprendre des analyses systématiques sur un grand nombre d'échantillons, il est nécessaire de déterminer les éléments qui parmi les plus aisés à doser seront les meilleurs ( indicateurs d'origine ).

Smith H. (1966) cité par Bryan D. E. (1967) sépare les traces contenues dans un échantillon biologique et donc dans une plante en deux grandes catégories. L'une groupe les éléments qui sont ou paraissent indispensables; leur concentration varie peu. L'autre comprend ceux qui ne semblent pas indispensables : ils présentent des distributions beaucoup plus larges. Autrement dit, la plante joue un rôle de c filtre " plus ou moins efficace vis-à-vis des éléments mis à sa disposition dans le sol, tandis qu'elle a peu ou pas d'action sur d'autres. La composition minérale du nectar qu'elle fournit et donc du miel qui en sera issu, est une image déformée de celle du sol : les éléments à faible taux de variation donneront une indication sur la nature des fleurs butinées, ceux à fort taux de variation renseigneront sur le sol et la provenance.

Sans parler des apports dus à l'abeille et à l'apiculteur, la composition minérale d'un miel peut être encore affectée par plusieurs facteurs et en particulier par la date de la récolte et par le climat, causes de variations dans la configuration florale du lieu ou du moment et, par suite, dans la proportion des différents nectars récoltés. Il faudra aussi vérifier que certains éléments ne sont pas introduits par une pollution industrielle ou agricole passagère ou encore par une quantité inhabituelle de pollen (VErmeulen L., 1965), ce qui serait le cas pour les miels de presse.

Si le sol contient un élément particulier et caractéristique, le miel en provenance de cette région sera ( marqué » et donc facilement reconnaissable. Sinon l'identification de l'origine nécessitera l'analyse de plusieurs éléments et la comparaison des résultats obtenus à ceux contenus dans un fichier central donnant pour chaque type de miel et chaque région d'origine les concentrations moyennes et limites que peuvent prendre ces éléments indicateurs.

L'importance du nombre d'échantillons à traiter, d'éléments à doser pour constituer ce fichier, nécessite de recourir à des méthodes d'analyse multiélémentaire fines ne demandant qu'un minimum de manipulations; tel est le cas de l'absorption atomique ou de l'analyse par activation. C'est précisément cette dernière technique qui est mise en œuvre dans le présent travail car elle a l'avantage de minimiser les pollutions toujours difficiles à éviter lorsqu'il s'agit de doser des traces et de ne requérir aucune séparation chimique pour un certain nombre d'éléments. 


\section{MATÉRIEL ET MÉTHODES}

Les propriétés physico-chimiques du miel sont telles qu'une irradiation directe ou une réduction en cendres sous pression réduite à l'aide d'oxygène atomique est impossible. Les échantillons de miel, $15 \mathrm{~g}$ environ, (pesés exactement) sont donc grillés lentement sous infrarouge dans une capsule en silice sans addition d'aucun réactif. Afin d'éviter la formation d'une mousse trop abondante on ajoute le miel par petites quantités au fur et à mesure du grillage à partir d'un flacon compte-gouttes en polyéthylène. Cette méthode est simple mais longue. La réduction totale en cendres n'a pas été recherchée, aussi les résultats sont-ils rapportés à une masse de miel frais. Les cendres obtenues sont transférées dans un tube en silice qui, une fois scellé, est irradié pendant 72 heures dans le flux de neutrons thermiques de la pile Pégase du Centre d'Études Nucléaires de Cadarache $\left(5.10^{13} \mathrm{n} . \mathrm{cm}^{-{ }^{2}}\right.$. sec-1). Une dizaine d'échantillons peuvent être irradiés simultanément.

Après irradiation les tubes sont laissés à “ refroidir ” pendant cinq jours environ a fin que disparaissent ou décroissent suffisamment les activités dues aux radioéléments de période courte (inférieure à une dizaine d'heures). Les tubes sont alors ouverts et les cendres transférées en totalité dans des capsules de comptage en polyéthylène.

Plusieurs spectrométries sont réalisées à l'aide d'usı diode Ge (Li) de $40 \mathrm{ml}$ associée à un sélecteur d'amplitude 4000 canaux “ Intertechnique \%. Elles sont réparties dans le temps pour atteindre aussi bien les radioéléments de période inférieure à quelques jours que ceux de période plus longue.

Chaque spectre transféré sur bande perforée est traité en ordinateur selon un programme de calcul (Panisset, 1973) qui gère les opérations suivantes : recherche des différentes raies gamma et détermination de leurs caractéristiques, attribution des activités correspondantes au temps origine. Pour effectuer ce travail deux fichiers sont stockés en machine : le premier regroupe les données relatives aux raies gamma des nucléides obtenues lors d'une irradiation en pile, le second les courbes d'efficacité du détecteur en fonction des énergies pour les différentes géométries de comptage.

Les spectres appartenant à un même échantillon, généralement au nombre de 4 , sont alors comparés de façon à éliminer les interférences possibles. Les activités retenues sont les moyennes des activités mesurées sous chaque pic pur issu d'un même radionucléide. Puisqu'il s'agit simplement de dégager les différences pouvant exister entre deux échantillons de miel il reste à comparer les activités obtenues, mais pour rendre expressifs les résultats, la conversion de ces dernières en unité de masse selon la relation suivante a paru préférable :

$$
\mathbf{M}=\mathbf{A}_{\mathbf{0}} \frac{\mathbf{A}_{\mathrm{t}} \cdot \mathbf{f} \cdot \mathbf{3}, 710^{4}}{\mathbf{\Phi} \cdot \sigma \cdot\left(1-\mathrm{e}^{-\lambda \mathrm{T}}\right) \cdot \mathscr{N}}
$$

La section efficace $\sigma$, la fraction isotopique $f$, la période $t_{1 / 2}=\frac{\text { Ln } 2}{\hbar}$ la masse atomique $A_{t}$ sont relevées dans la littérature (Junod E., 1970, Koch R. O., 1960); le flux $\Phi$ est calculé à partir de l'activité d'une quantité connue de fer irradié en même temps que l'échantillon. $\mathscr{N}$ est le nombre d'Avogadro et $T$ le temps d'irradiation. $A_{0}$ représente l'activité au temps origine c'est-à-dire à la fin de l'irradiation. L'imprécision dans la connaissance de certaines constantes comme la section efficace peut entraîner une erreur systématique par rapport à la masse vraie mais cette erreur, gênante dans le cas d'un dosage quantitatif, ne l'est pas lorsque les échantillons sont comparés entre eux.

\section{RÉSULTATS}

Les origines géographiques des différents miels étudiés sont données dans le tableau 1 (Des analyses polliniques et chimiques ont permis de vérifier la qualité de ces miels unifloraux). Les tableaux 2 à 5 résument les concentrations calculées selon la formule précédente : elles s'échelonnent de 8.10-3 g à 5.10-9 g. par gramme de miel frais. 
TaBL. 1. - Origine des différents miels êtudiés.

TAB. I. - Herkunft der verschiedenen untersuchten Honige

\section{MIELS CLAIRS}

Helle Honige

\begin{tabular}{|c|c|c|c|}
\hline No & $\begin{array}{c}\text { Acacia } \\
\text { (Robinia pseudacacia) }\end{array}$ & No & $\begin{array}{l}\text { Lavande et Lavandin } \\
\quad(\text { Lavandula) }\end{array}$ \\
\hline 1 & Hongrie & 14 & $\begin{array}{l}\text { Vaucluse. La Gabelle (Massif du mont } \\
\text { Ventoux) }\end{array}$ \\
\hline 3 & Bas-Rhin & 15 & Vaucluse. Région de Valréas \\
\hline 4 & Loir-et-Cher & 16 & $\begin{array}{l}\text { Alpes de Haute Provence. } \\
\text { (Rémoules/Ségries) }\end{array}$ \\
\hline 5 & Haute-Saône & & \\
\hline 6 & Gironde (Vallée de la Garonne-Sud) & & \\
\hline
\end{tabular}

MIELS FONCÉS

Dunkle Honige

\begin{tabular}{|c|c|c|c|}
\hline No & $\begin{array}{c}\text { Sapin } \\
\text { (Abies pectinata) }\end{array}$ & $\mathrm{No}^{\circ}$ & $\begin{array}{l}\text { Bruyère (Callune) } \\
\text { (Calluna vulgaris) }\end{array}$ \\
\hline 7 & Loire & 11 & Landes (Étang de Léon - Bord de mer) \\
\hline 8 & Alsace (Vallée de Munster) & 12 & Landes (Région de Sabres - Grande Lande) \\
\hline 9 & Vosges (Raon l'Étape) & 13 & Cévennes (Col du Mas de l'Air) \\
\hline 10 & Vosges (Ballon d'Alsace) & & \\
\hline
\end{tabular}


TABL. 2. - Concentration des élêments dans les miels d'acacia (Robinia pseudacacia) (:^g/g) $\simeq$ pic faible : estimation de la concentration $<$ pic non vu : limite supérieure

TAB. 2. - Konzentration der Elemente in den Akazienhonigen (Robinia pseudacacia ( $\mu \mathrm{g} / \mathrm{g}$ ) $\simeq$ schwacher Pik $=$ geschätzte Konzentration

$<$ Pik nicht gesehen $=$ oberste Grenze

\begin{tabular}{|c|c|c|c|c|c|}
\hline & Hongrie. 1 & Bas-Rhin. 3 & Loir-et-Cher. 4 & Haute-Saône. 5 & Gironde. 6 \\
\hline $\mathbf{K}$. & $0,82.10^{+2}$ & $0,14 \cdot 10^{+3}$ & $0,73 \cdot 10^{+2}$ & $0,76 \cdot 10^{+2}$ & $0,64 \cdot 10^{+2}$ \\
\hline $\mathrm{Ca}$ & $0,13 \cdot 10^{+2}$ & $0,99 \cdot 10^{+1}$ & $0,32.10^{+1}$ & $0,10.10^{+2}$ & $0,85 \cdot 10^{+1}$ \\
\hline $\mathbf{N a}$ & $0,27 \cdot 10^{+1}$ & $0,16 \cdot 10^{+1}$ & $0,78 \cdot 10^{+0}$ & $0,14 \cdot 10^{+1}$ & $0,15 \cdot 10^{+1}$ \\
\hline $\mathrm{Zn}$ & $0,12.10^{+1}$ & $0,43 \cdot 10^{+0}$ & $0,99.10^{+0}$ & $0,52.10^{+0}$ & $0,15 \cdot 10^{+0}$ \\
\hline $\mathbf{R b}$ & $0,46 \cdot 10^{-1}$ & $0,30 \cdot 10^{+0}$ & $0,19.10^{+0}$ & $0,13 \cdot 10^{+0}$ & $0,67 \cdot 10^{-1}$ \\
\hline $\mathrm{Fe}$ & $0,51 \cdot 10^{+0}$ & $0,53 \cdot 10^{+0}$ & $0,81 \cdot 10^{+0}$ & $0,25 \cdot 10^{+0}$ & $0,53 \cdot 10^{+0}$ \\
\hline Sn & $0,82,10^{-1}$ & $0,10 \cdot 10^{-1}$ & $0,12.10^{+0}$ & $0,60.10^{-1}$ & $0,85 \cdot 10^{-1}$ \\
\hline $\mathbf{B a}$ & $0,22 \cdot 10^{-1}$ & $\simeq 0,90 \cdot 10^{-2}$ & $0,50.10^{-2}$ & $<0,60.10^{-2}$ & $<0,50.10^{-2}$ \\
\hline $\mathbf{B r}$ & $0,19 \cdot 10^{-1}$ & $0,28 \cdot 10^{-1}$ & $0,86 \cdot 10^{-2}$ & $0,86 \cdot 10^{-2}$ & $0,88 \cdot 10^{-2}$ \\
\hline $\mathbf{N i}$ & $0,11 \cdot 10^{-2}$ & $0,44 \cdot 10^{-2}$ & $0,32.10^{-2}$ & $0,28 \cdot 10^{-2}$ & $0,78 \cdot 10^{-3}$ \\
\hline $\mathrm{Cr}$ & $0,55 \cdot 10^{-2}$ & $0,43 \cdot 10^{-2}$ & $0,60 \cdot 10^{-3}$ & $0,17 \cdot 10^{-1}$ & $0,20 \cdot 10^{-3}$ \\
\hline Cs & $0,10.10^{-3}$ & $0,95.10^{-2}$ & $0,19 \cdot 10^{-2}$ & $0,44.10^{-2}$ & $0,20 \cdot 10^{-3}$ \\
\hline $\mathbf{L a}$ & $0,21 \cdot 10^{-3}$ & $0,21.10^{-3}$ & $0,46.10^{-3}$ & $0,15 \cdot 10^{-3}$ & $0,12 \cdot 10^{-3}$ \\
\hline Co & $0,47 \cdot 10^{-3}$ & $0,75 \cdot 10^{-3}$ & $0,50.10^{-3}$ & $0,22.10^{-3}$ & $0,49 \cdot 10^{-3}$ \\
\hline $\mathrm{Sb}$ & $0,30.10^{-4}$ & $0,13 \cdot 10^{-3}$ & $0,33.10^{-3}$ & $0,10 \cdot 10^{-3}$ & $0,11 \cdot 10^{-3}$ \\
\hline $\mathrm{Sc}$ & $0,43 \cdot 10^{-4}$ & $0,32 \cdot 10^{-4}$ & $0,52 \cdot 10^{-4}$ & $0,80 \cdot 10^{-5}$ & $0,46.10^{-4}$ \\
\hline$A u$ & $0,35 \cdot 10^{-5}$ & $0,80 \cdot 10^{-5}$ & $0,61 \cdot 10^{-5}$ & $0,98 \cdot 10^{-5}$ & $0,30.10^{-6}$ \\
\hline Yb & & & & & \\
\hline Hf & & $0,70 \cdot 10^{-4}$ & $0,15 \cdot 10^{-3}$ & & \\
\hline
\end{tabular}


TABL. 3. - Concentration des éléments dans les miels de lavande et lavandin (Lavandula) ( $\mu \mathrm{g} / \mathrm{g}$ )

TAB. 3. - Konzentration der Elemente in den Lavendel - und Lavandinhonigen (Lavandula) ( $\mu \mathrm{g} / \mathrm{g}$ )

\begin{tabular}{|c|c|c|c|}
\hline & Lavande & \multicolumn{2}{|c|}{ Lavandin } \\
\hline & La Gabelle. I4 & Valréas. 15 & Remoules. 16 \\
\hline $\mathbf{K}$ & $0,31 \cdot 10^{+3}$ & $0,21.10^{+3}$ & $0,37 \cdot 10^{+3}$ \\
\hline $\mathbf{C a}$ & $0,66.10^{+2}$ & $0,98.10^{+2}$ & $0,11 \cdot 10^{+3}$ \\
\hline $\mathbf{N a}$ & $0,68 \cdot 10^{+1}$ & $0,69 \cdot 10^{+1}$ & $0,11 \cdot 10^{+2}$ \\
\hline$Z \mathbf{n}$ & $0,36 \cdot 10^{+0}$ & $0,23 \cdot 10^{+1}$ & $0,49.10^{+0}$ \\
\hline $\mathbf{R b}$ & $0,14.10^{+0}$ & $0,15 \cdot 10^{+0}$ & $0,17 \cdot 10^{+0}$ \\
\hline $\mathrm{Fe}$ & $0,64 \cdot 10^{+0}$ & $0,13 \cdot 10^{+1}$ & $0,22.10^{+1}$ \\
\hline Sn & $0,16 \cdot 10^{+0}$ & $\simeq 0,40.10^{-1}$ & $0,47 \cdot 10^{+0}$ \\
\hline $\mathbf{B a}$ & $0,17 \cdot 10^{-1}$ & $0,90 \cdot 10^{-1}$ & $0,17.10^{-1}$ \\
\hline $\mathrm{Br}$ & $0,25 \cdot 10^{-1}$ & $0,25 \cdot 10^{-1}$ & $0,67,10^{-1}$ \\
\hline $\mathrm{Ni}$ & $<0,10 \cdot 10^{-2}$ & $\simeq 0,30 \cdot 10^{-2}$ & $\simeq 0,20.10^{-2}$ \\
\hline $\mathrm{Cr}$ & $0,14 \cdot 10^{-1}$ & $0,55.10^{-1}$ & $0,12.10^{-1}$ \\
\hline $\mathrm{Cs}$ & $0,63 \cdot 10^{-3}$ & $0,84 \cdot 10^{-3}$ & $0,56 \cdot 10^{-3}$ \\
\hline $\mathrm{La}$ & $0,51 \cdot 10^{-3}$ & $0,39 \cdot 10^{-2}$ & $0,18.10^{-2}$ \\
\hline Co & $0,12 \cdot 10^{-2}$ & $0,12 \cdot 10^{-2}$ & $0,24.10^{-2}$ \\
\hline $\mathrm{Sb}$ & $0,38 \cdot 10^{-1}$ & $0,18 \cdot 10^{-2}$ & $0,11.10^{-2}$ \\
\hline $\mathrm{Se}$ & $0,12 \cdot 10^{-3}$ & $0,34 \cdot 10^{-3}$ & $0,31 \cdot 10^{-3}$ \\
\hline $\mathrm{Au}$ & $\simeq 0,60 \cdot 10^{-5}$ & $0,24 \cdot 10^{-4}$ & $0,10.10^{-4}$ \\
\hline $\mathrm{Hg}$ & $0,11 \cdot 10^{-2}$ & $0,31 \cdot 10^{-2}$ & $0,28 \cdot 10^{-2}$ \\
\hline $\mathbf{H f}$ & $0,10 \cdot 10^{-3}$ & $0,20 \cdot 10^{-2}$ & $0,60 \cdot 10^{-3}$ \\
\hline $\mathbf{Y b}$ & & $0,40 \cdot 10^{-2}$ & $0,60 \cdot 10^{-2}$ \\
\hline Ta & & $0,70 \cdot 10^{-4}$ & $0,40.10^{-4}$ \\
\hline $\mathrm{Tb}$ & & $0,80 \cdot 10^{-4}$ & \\
\hline $\mathrm{Zr}$ & & $0,30 \cdot 10^{-1}$ & \\
\hline
\end{tabular}


TaBL. 4. - Concentration des élêments dans les miels de callune (Calluna vulgaris) $\mu \mathrm{g} . / \mathrm{g}$.

TAв. 4. - Konzentration der Elemente in den Heidehonigen (Calluna vulgaris) (ig./g.)

\begin{tabular}{|c|c|c|c|}
\hline & Êtang de Léon. 11 & Région de Sabres. 12 & Cévennes. 13 \\
\hline $\begin{array}{l}\mathrm{K} \\
\mathrm{Ca} \\
\mathrm{Na} \\
\mathrm{Zn} \\
\mathrm{Rb} \\
\mathrm{Fe} \\
\mathrm{Sn} \\
\mathrm{Ba} \\
\mathrm{Br} \\
\mathrm{Ni} \\
\mathrm{Cr} \\
\mathrm{Cs} \\
\mathrm{La} \\
\mathrm{Co} \\
\mathrm{Sb} \\
\mathrm{Sc} \\
\mathrm{Au}\end{array}$ & $\begin{array}{r}0,70 \cdot 10^{+4} \\
0,24 \cdot 10^{+3} \\
0,15 \cdot 10^{+3} \\
0,99 \cdot 10^{+0} \\
0,53 \cdot 10^{+1} \\
0,91 \cdot 10^{+0} \\
<0,10 \cdot 10^{+0} \\
0,30 \cdot 10^{+0} \\
0,27 \cdot 10^{+0} \\
\simeq 0,10 \cdot 10^{-1} \\
0,44 \cdot 10^{-2} \\
0,25 \cdot 10^{+0} \\
\simeq 0,30 \cdot 10^{-3} \\
0,64 \cdot 10^{-3} \\
0,56 \cdot 10^{-3} \\
<0,10 \cdot 10^{-4} \\
0,22 \cdot 10^{-3}\end{array}$ & $\begin{array}{r}0,72 \cdot 10^{+4} \\
0,27 \cdot 10^{+3} \\
0,13 \cdot 10^{+3} \\
0,48 \cdot 10^{+0} \\
0,63 \cdot 10^{+1} \\
0,78 \cdot 10^{+0} \\
<0,10 \cdot 10^{+0} \\
0,36 \cdot 10^{+0} \\
0,18 \cdot 10^{+0} \\
<0,60 \cdot 10^{-2} \\
0,92 \cdot 10^{-2} \\
0,41 \cdot 10^{+0} \\
\simeq 0,20 \cdot 10^{-3} \\
0,42 \cdot 10^{-3} \\
0,60 \cdot 10^{-3} \\
<0,10 \cdot 10^{-4} \\
0,28 \cdot 10^{-3}\end{array}$ & $\begin{array}{r}0,65 \cdot 10^{+4} \\
0,28 \cdot 10^{+3} \\
0,61 \cdot 10^{+2} \\
0,25 \cdot 10^{+1} \\
0,37 \cdot 10^{+1} \\
0,15 \cdot 10^{+1} \\
<0,10 \cdot 10^{+0} \\
0,25 \cdot 10^{+0} \\
0,16 \cdot 10^{+0} \\
<0,60 \cdot 10^{-2} \\
0,19 \cdot 10^{-1} \\
0,17 \cdot 10^{+0} \\
0,17 \cdot 10^{-2} \\
0,12 \cdot 10^{-2} \\
0,11 \cdot 10^{-2} \\
0,17 \cdot 10^{-3} \\
0,10 \cdot 10^{-3}\end{array}$ \\
\hline
\end{tabular}

TaBL. 5. - Concentration des éléments dans les miels de miellat de Sapin (Abies pectinata) ( $\mu \mathrm{g} . / \mathrm{g}$.

TAB. 5. - Konzentration der Elemente in den Honigtauhonigen der Tanne (Abies pectinata) ( $\mu \mathrm{g} . / \mathrm{g}$.

\begin{tabular}{|c|c|c|c|c|}
\hline & Loire. 7 & Vallée de Munster. 8 & Raon 1'Étape. 9 & Ballon d'Alsace. 10 \\
\hline $\begin{array}{l}\mathrm{K} \\
\mathrm{Ca} \\
\mathrm{Na} \\
\mathrm{Zn} \\
\mathrm{Rb} \\
\mathrm{Fe} \\
\mathrm{Sn} \\
\mathrm{Ba} \\
\mathrm{Br} \\
\mathrm{Ni} \\
\mathrm{Cr} \\
\mathrm{Cs} \\
\mathrm{La} \\
\mathrm{Co} \\
\mathrm{Sb} \\
\mathrm{Sc} \\
\mathrm{Au} \\
\mathrm{Ag}\end{array}$ & $\begin{array}{r}0,59 \cdot 10^{+4} \\
<0,16 \cdot 10^{+2} \\
0,11 \cdot 10^{+2} \\
0,23 \cdot 10^{+1} \\
0,38 \cdot 10^{+2} \\
0,12 \cdot 10^{+2} \\
<0,30 \cdot 10^{+0} \\
<0,50 \cdot 10^{+0} \\
0,16 \cdot 10^{+0} \\
0,52 \cdot 10^{-1} \\
0,26 \cdot 10^{-1} \\
0,17 \cdot 10^{+1} \\
0,25 \cdot 10^{-2} \\
0,46 \cdot 10^{-1} \\
0,83 \cdot 10^{-2} \\
0,47 \cdot 10^{-3} \\
0,22 \cdot 10^{-3} \\
0,37 \cdot 10^{-1}\end{array}$ & $\begin{array}{r}0,78 \cdot 10^{+4} \\
\simeq 0,37 \cdot 10^{+2} \\
0,73 \cdot 10^{+1} \\
0,22 \cdot 10^{+1} \\
0,17 \cdot 10^{+2} \\
0,57 \cdot 10^{+1} \\
0,65 \cdot 10^{+0} \\
<0,30 \cdot 10^{+0} \\
0,18 \cdot 10^{+0} \\
0,81 \cdot 10^{-1} \\
0,28 \cdot 10^{-1} \\
0,66 \cdot 10^{+0} \\
0,18 \cdot 10^{-2} \\
0,48 \cdot 10^{-1} \\
0,42 \cdot 10^{-2} \\
0,54 \cdot 10^{-3} \\
\simeq 0,70 \cdot 10^{-4} \\
\simeq 0,20 \cdot 10^{-2}\end{array}$ & $\begin{aligned} & 0,41 \cdot 10^{+4} \\
& \simeq 0,32 \cdot 10^{+2} \\
& 0,13 \cdot 10^{+2} \\
& 0,84 \cdot 10^{+1} \\
& 0,11 \cdot 10^{+2} \\
& 0,11 \cdot 10^{+2} \\
& \simeq 0,20 \cdot 10^{+0} \\
&<0,30 \cdot 10^{+0} \\
& 0,22 \cdot 10^{+0} \\
& 0,80 \cdot 10^{-1} \\
& 0,22 \cdot 10^{-1} \\
& 0,21 \cdot 10^{+0} \\
& 0,16 \cdot 10^{-2} \\
& 0,12 \cdot 10^{-1} \\
& 0,41 \cdot 10^{-2} \\
& 0,40 \cdot 10^{-3} \\
& \simeq 0,40 \cdot 10^{-4} \\
&<0,10 \cdot 10^{-2}\end{aligned}$ & $\begin{array}{r}0,52 \cdot 10^{+4} \\
0,12 \cdot 10^{+3} \\
0,11 \cdot 10^{+2} \\
0,18 \cdot 10^{+2} \\
0,19 \cdot 10^{+2} \\
0,57 \cdot 10^{+1} \\
<0,20 \cdot 10^{+0} \\
<0,40 \cdot 10^{+0} \\
0,12 \cdot 10^{+0} \\
0,81 \cdot 10^{-1} \\
0,43 \cdot 10^{-1} \\
0,15 \cdot 10^{+1} \\
0,19 \cdot 10^{-2} \\
0,19 \cdot 10^{-1} \\
0,18 \cdot 10^{-2} \\
0,38 \cdot 10^{-3} \\
<0,20 \cdot 10^{-4} \\
<0,10 \cdot 10^{-2}\end{array}$ \\
\hline
\end{tabular}


L'analyse spectrométrique directe c'est-à-dire sans aucune séparation chimique, telle qu'elle a été réalisée au cours de ce travail, présente de nombreux avantages. Elle ne permet pas cependant le dosage simultané de tous les éléments : concentrations trop faibles ou caractéristiques nucléaires défavorables (période, énergie gamma, rendement...). Par exemple dans les conditions choisies, il est impossible de doser le manganèse et le chlore en raison de leurs trop courtes périodes; le cuivre, émetteur d'une seule raie à $511 \mathrm{KeV}$, ne peut être mesuré à cause de nombreuses interférences à cette énergie.

Entre possibilité et impossibilité de dosage existent deux cas particuliers: - un pic émerge au-dessus du bruit de fond mais sa surface est trop faible pour permettre un dosage : la valeur de la masse donnée dans les tableaux 2 à 5 n'est qu'une estimation, elle est précédée du signe $\simeq$.

- aucun pic n'émerge du fond continu mais la comparaison avec les autres échantillons montre que l'élément est probablement présent : la valeur notée est alors une limite supérieure, elle est précédée du signe < qui signifie NON VU.

Dans tous ces cas, d'autres conditions d'analyses ou une séparation chimique après irradiation serait nécessaire pour effectuer le dosage.

Les diagrammes ou “ spectres élémentaires » (fig. 1 à 4) sont obtenus en portant en abscisse les éléments dosés par ordre d'importance moyenne décroissante et en ordonnée les concentrations normalisées de telle sorte que la masse totale des traces dosées soit de $1000 \mathrm{ppm}$. Cette normalisation équivaut à rapporter l'ensemble des masses à une masse de potassium (élément de loin le plus important) constante. Elle n'a d'autre ambition que de faciliter la comparaison des différents « spectres ».

\section{DISCUSSION}

Bien qu'il soit aisé de distinguer un miel “ clair " d'un miel “ foncé 》, un miel d'acacia d'un autre de lavande sans faire appel à des techniques complexes, il est intéressant de noter comment ces différences se traduisent au niveau des traces minérales avant de tenter une recherche de l'origine géographique.

\section{1. - Distinction entre miel clair et miel foncé}

Quelques constatations peuvent être faites sur ces deux types de miel sans cependant s'attarder à chercher des critères de distinction puisqu'un coup d'ceil suffit. Les moyennes et les limites atteintes par les concentrations d'une 

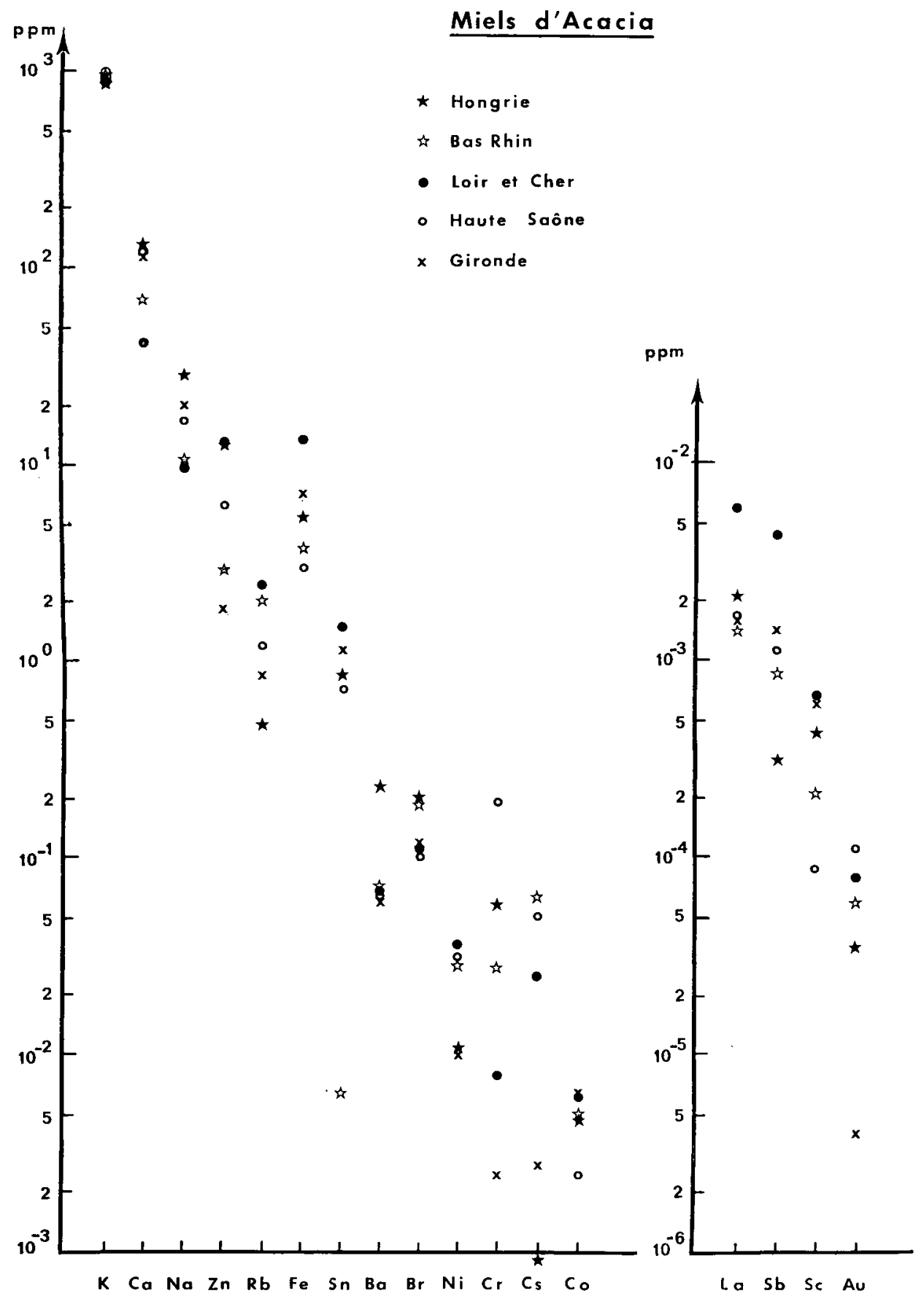

FIG. 1 

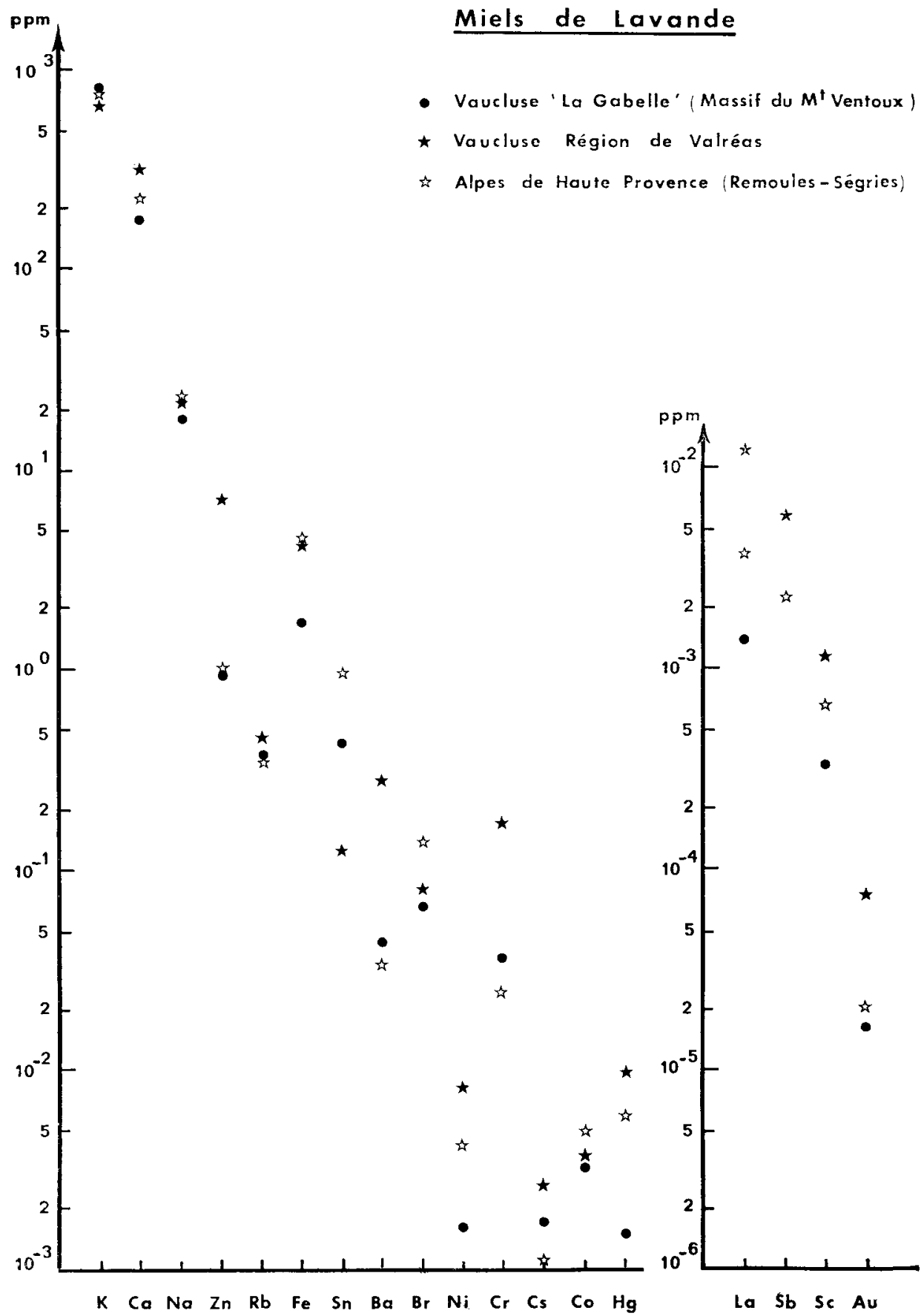

FIG. 2 


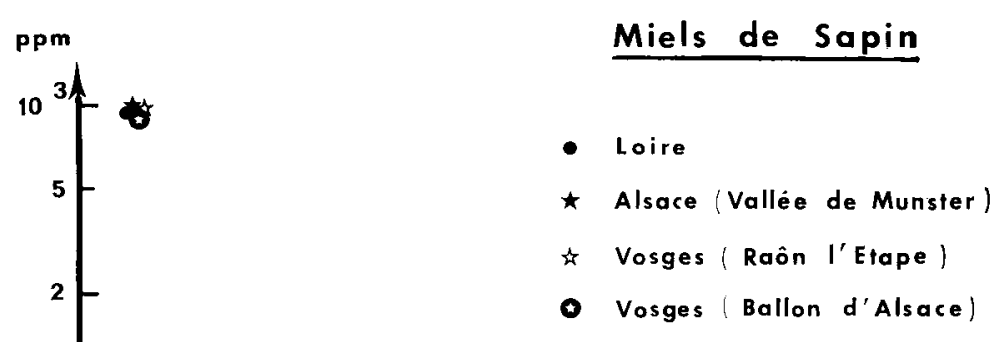

10
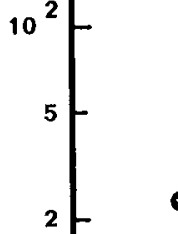

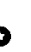

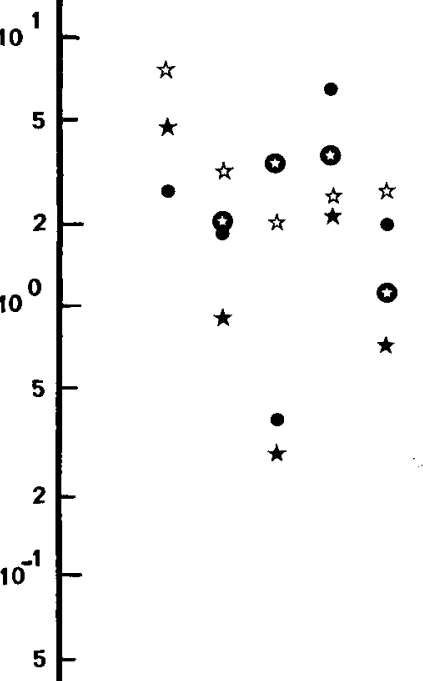

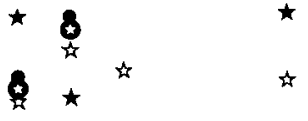

Ppm

$\$$

2

$10^{-2}$

0

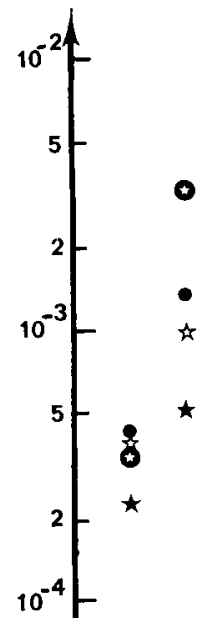

$0^{-3}$

K Ca Na Zn Rb Fe Sn Ba Br Ni Cr Cs, Co Ag

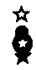

-

Fig. 3 
ppm

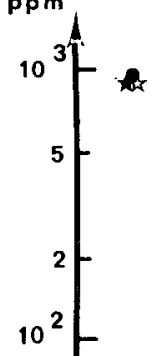

\section{Miels de Callune}

* Landes Etang de Léon, Bord de Mer

Landes Région de Sabres, Grande Lande

- Cévennes Col du mas de l'air

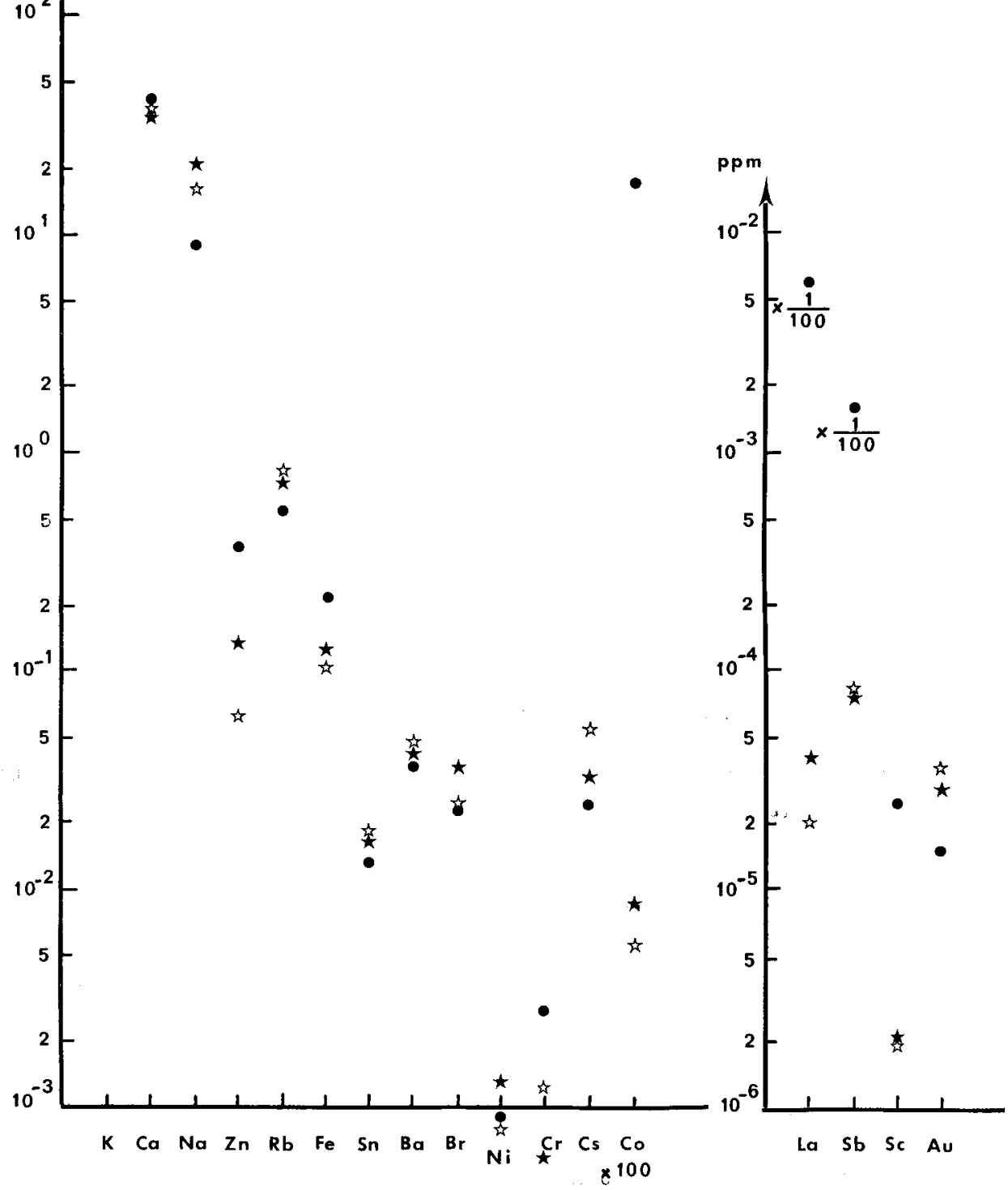

FIG. 4 
TABL. 6. - Concentrations moyennes et limites (ppm).

TAB. 6. - Mittlere und Grenzwertkonzentrationen (ppm).

\begin{tabular}{|c|c|c|c|c|c|c|}
\hline \multirow[b]{2}{*}{ Éléments } & \multicolumn{3}{|c|}{$\begin{array}{c}\text { MIELS CLAIRS } \\
\text { Helle Honige }\end{array}$} & \multicolumn{3}{|c|}{$\begin{array}{c}\text { MIELS FONGÉS } \\
\text { Dunkle Honige }\end{array}$} \\
\hline & Moyenne & Minimum & Maximum & Moyenne & Minimum & Maximum \\
\hline $\begin{array}{l}\mathrm{K} \\
\mathrm{Ca} \\
\mathrm{Na} \\
\mathrm{Rb} \\
\mathrm{Cs} \\
\mathrm{Ba} \\
\mathrm{Br} \\
\mathrm{Fe} \\
\mathrm{Zn} \\
\mathrm{Co} \times 10^{-3} \\
\mathrm{Cr} \times 10^{-3}\end{array}$ & $\begin{array}{c}165 \\
40 \\
4 \\
0,15 \\
0,002 \\
0,02 \\
0,024 \\
0,84 \\
0,8 \\
0,9 \\
12\end{array}$ & $\begin{array}{l}66 \\
3,2 \\
0,8 \\
0,05 \\
0,0001 \\
0,005 \\
0,009 \\
0,25 \\
0,15 \\
0,2 \\
0,2\end{array}$ & $\begin{array}{c}368 \\
107 \\
11 \\
0,3 \\
0,010 \\
0,09 \\
0,067 \\
2,2 \\
2,3 \\
2,4 \\
55\end{array}$ & $\begin{array}{c}6250 \\
142 \\
55 \\
14 \\
0,7 \\
0,33 \\
0,18 \\
5,4 \\
5 \\
18 \\
19\end{array}$ & $\begin{array}{cl}4 & 130 \\
16 & \\
& 7,3 \\
& 3,7 \\
& 0,17 \\
& 0,3 \\
& 0,12 \\
& 0,8 \\
0,48 \\
0,4 \\
4\end{array}$ & $\begin{array}{c}7810 \\
280 \\
152 \\
38 \\
1,7 \\
0,4 \\
0,27 \\
12 \\
18 \\
48 \\
43\end{array}$ \\
\hline
\end{tabular}

douzaine d'éléments donnés dans le tableau 6 sont comparables à celles trouvées dans la littérature, bien que légèrement plus faibles, (Petrov V., 1970). Elles montrent que les domaines de variation des alcalins, du baryum et du brôme sont essentiellement différents et qu'il est toujours possible de distinguer les deux types de miels à l'aide de l'un ou de plusieurs d'entre eux. La figure 5 schématise les écarts pour les alcalins et les alcalino-terreux mesurés.

Les rapports $\mathrm{Rb} / \mathrm{Fe}$ et $\mathrm{Rb} / \mathrm{Zn}$ sont également caractéristiques des deux types de miels : inférieurs à 1 pour les miels clairs, ils lui deviennent supérieurs ou égaux pour les miels foncés (tableau 7).

\section{2. - Miels clairs. Distinction entre Acacia et Lavande.}

Le miel de lavande est dans l'ensemble plus riche en alcalins que celui d'acacia sauf en rubidium et en césium, tandis que le chrome, le fer, le cobalt, le nickel et le zinc sont dans des concentrations voisines (fig. 6). Le mercure présent en quantité relativement abondante dans le miel de lavande, n'est pas vu dans celui d'acacia $(2,3,10-3 \mu \mathrm{g})$. Cette trace particulière peut provenir soit du sol et dans ce cas constituer un excellent (c indicateur d'origine », soit être dû à une pollution industrielle ou agricole passagère interdisant son utilisation comme traceur.

\section{3. - Miels foncés, distinction entre Sapin et Callune}

Sauf pour le rubidium, les différences de concentration pour les alcalins et les alcalino-terreux sont à peu près identiques à celles qui existent entre les 


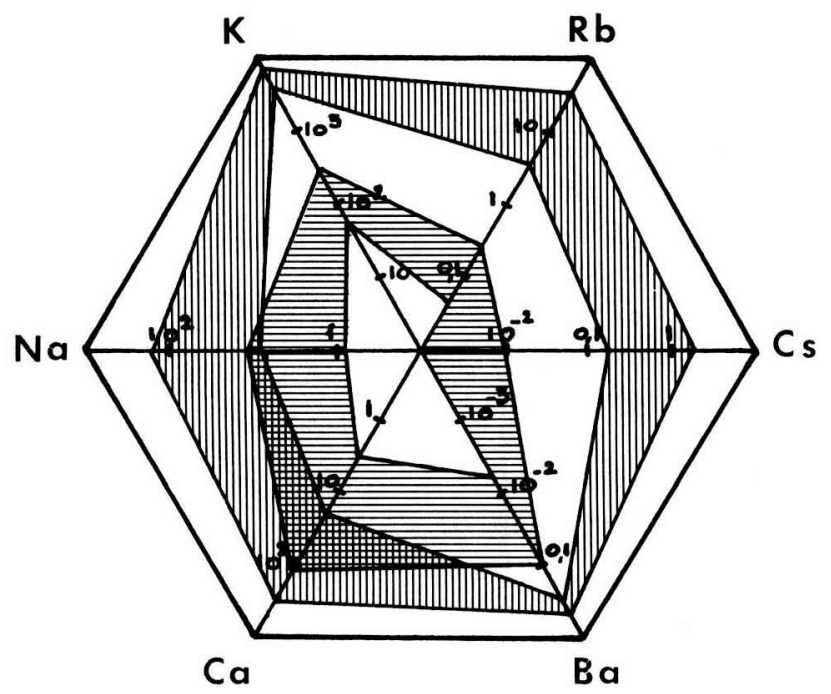

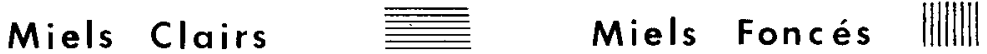

Fig. 5 -Différence entre miels clairs et miels foncés. Limites inférieures et supérieures (ppm) atteintes par 6 éléments dans les miels clairs et les miels foncés.

Aв8. 5 - Unterschied zwischen hellen und dunklen Honigen. Untere und obere Grenzen (ppm), die von 6 Elementen in hellen und dunklen Honigen erreieht wurden

miels clairs; par contre les écarts sont beaucoup plus significatifs pour le fer, le cobalt et le nickel (fig. 7).

\section{4. - Miels de même espèce, distinction des origines}

Tous les miels analysés possèdent les mêmes traces. La reconnaissance des origines ne peut done se faire par l'identification d'un seul et unique élément sauf pour quelques exceptions telles que le miel de sapin de Haute-Loire grâce à l'argent. La comparaison des rapports de concentration atteints par quelques éléments apporte d'autres informations. Ainsi en observant le tableau 7, on constate que les rapports $\mathrm{Rb} / \mathrm{Fe}$ et $\mathrm{Rb} / \mathrm{Zn}$ différencient le miel de Hongrie des autres miels d'acacia français. De même, le rapport $\mathrm{Rb} / \mathrm{Zn}$ est nettement plus faible pour le lavandin de plaine de Valréas, que pour les deux lavandes de montagne; à l'inverse il est plus fort et semble séparer, avec un degré de certitude moindre, le miellat de Loire des autres miellats alsaciens.

Enfin l'attribution d'une origine à tous les autres échantillons ne pourra se faire que par la comparaison des concentrations atteintes par plusieurs éléments à un catalogue de référence. Cette comparaison ne sera possible que dans la mesure où, pour un lieu de récolte donné, les teneurs de ces éléments resteront comprises entre deux limites quelle que soit l'année de la récolte. 


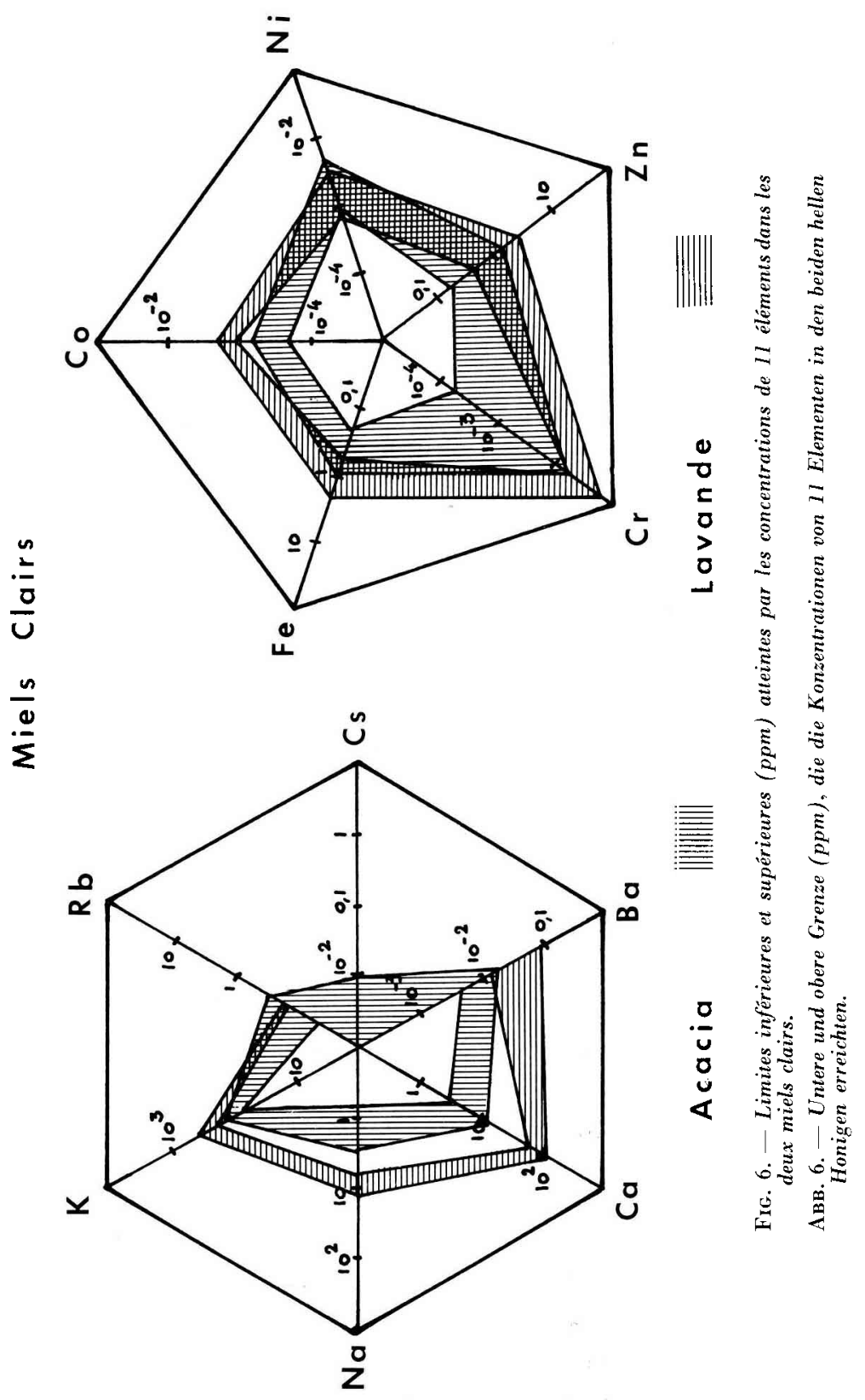




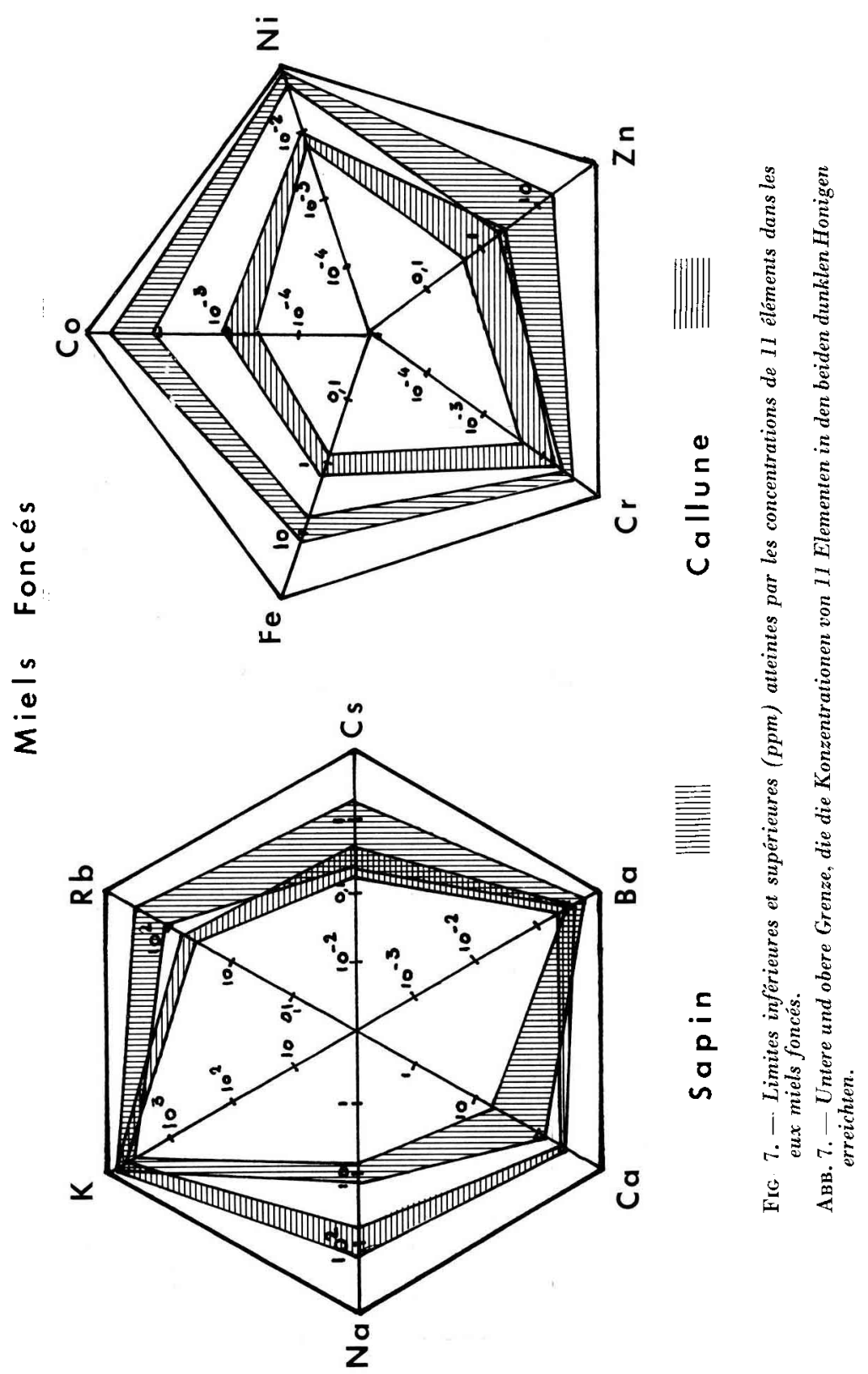




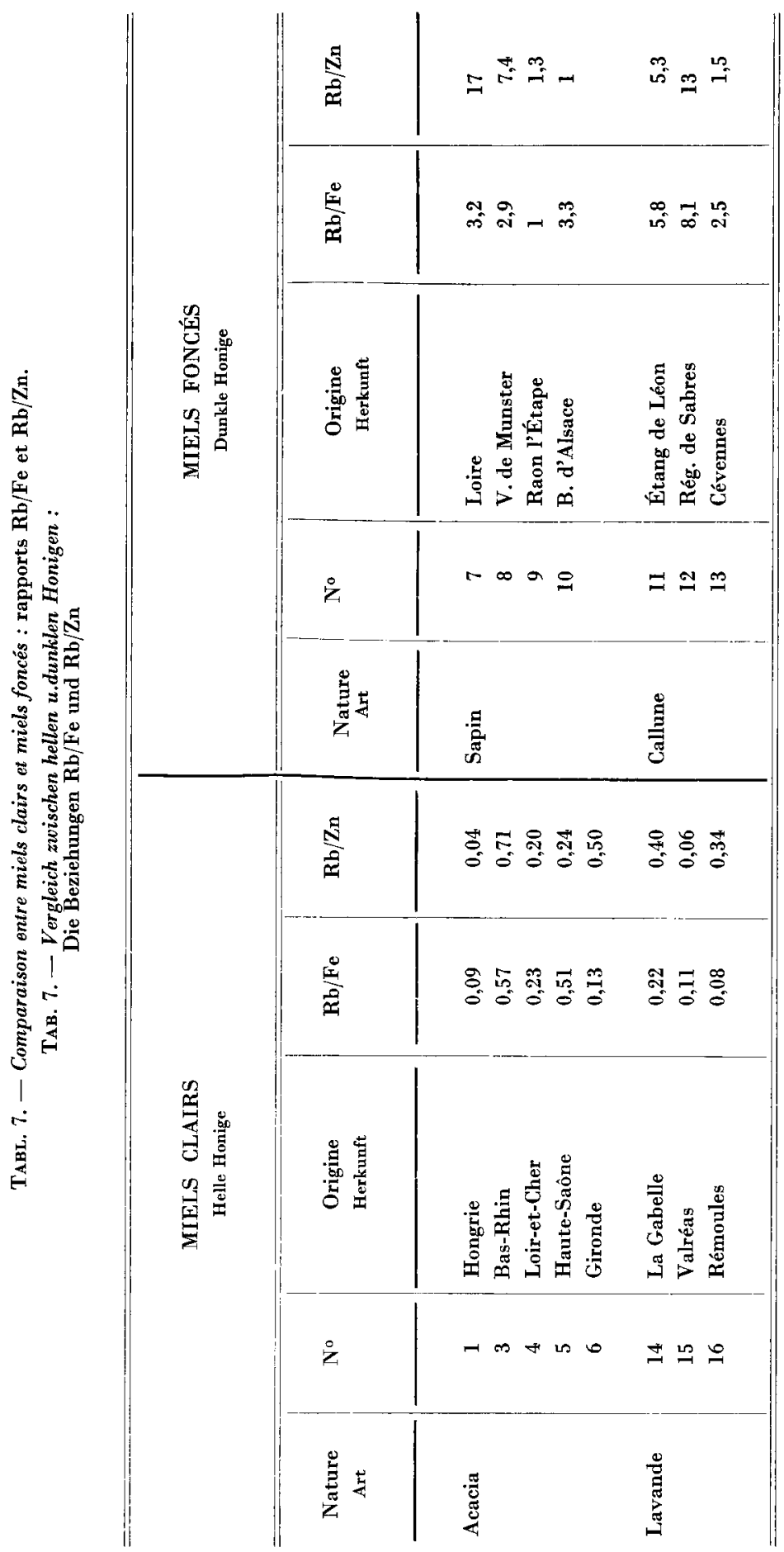


La détermination de ces fourchettes exige l'analyse de nombreux échantillons.

Le tri des miels étudiés à l'occasion de ce travail en fonction de leurs origines géographiques peut être réalisé en considérant que les teneurs trouvées pour chaque élément sont des teneurs moyennes et que tous les autres échantillons en provenance du même endroit auront les mêmes teneurs à un certain pourcentage de variation près.

Ce pourcentage qui doit tenir compte aussi bien des variations climatiques annuelles, des écarts introduits par les différents apiculteurs travaillant sur le même site, que des erreurs d'analyse, ne sera pas en réalité identique pour tous les éléments.

Les tableaux 8 et 9 ci-après résument les résultats obtenus après deux tris selon deux critères. Dans le premier cas on suppose qu'il y a même origine si les concentrations de l'élément considéré sont égales à $50 \%$ près c'est-à-dire si le rapport des deux valeurs est inférieur à 3 . Dans le second cas, la marge d'erreur est portée à $66,6 \%$ soit un rapport de 5 .

Dans le tableau 8, donné à titre d'exemple, chaque miel d'acacia est comparé aux autres miels d'acacia élément par élément. Pour le potassium

TABL. 8. - Distinction des origines pour les miels d'Acacia

TAB. 8. - Unterscheidung der Herkunft bei Akazienhonigen

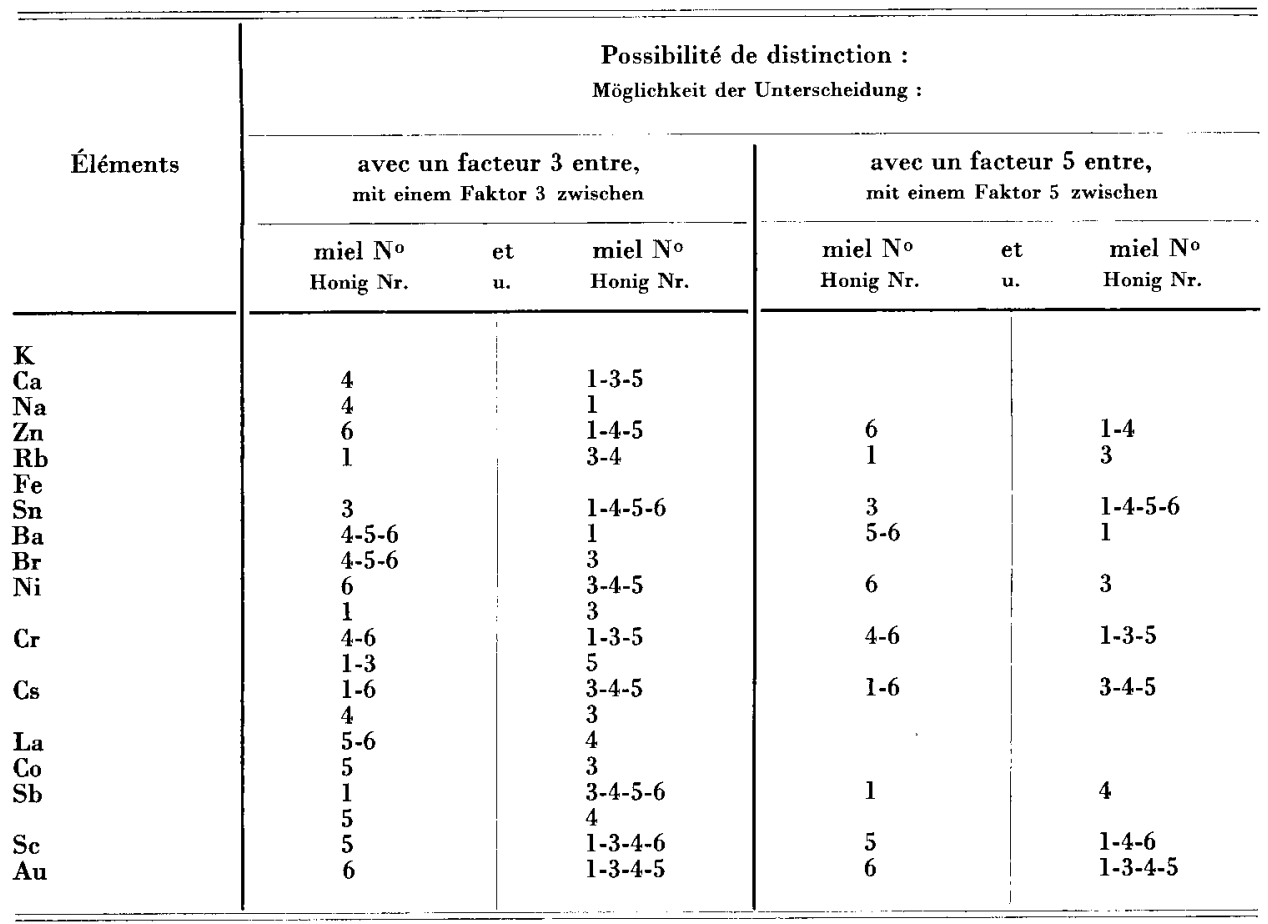


TABL. 9. - Distinction des origines

Les miels de la colonne I sont différents des miels de la colonne II par les éléments de la colonne III (coefficient 3) et de la colonne IV (coefficient 5)

TaB. 9. - Unterscheidung der Herkunft

Die Honige der Spalte I unterscheiden sich von denen der Spalte II durch die Elemente d. Spalte III (Koeffizient 3) u. IV (Koeffizient 5)

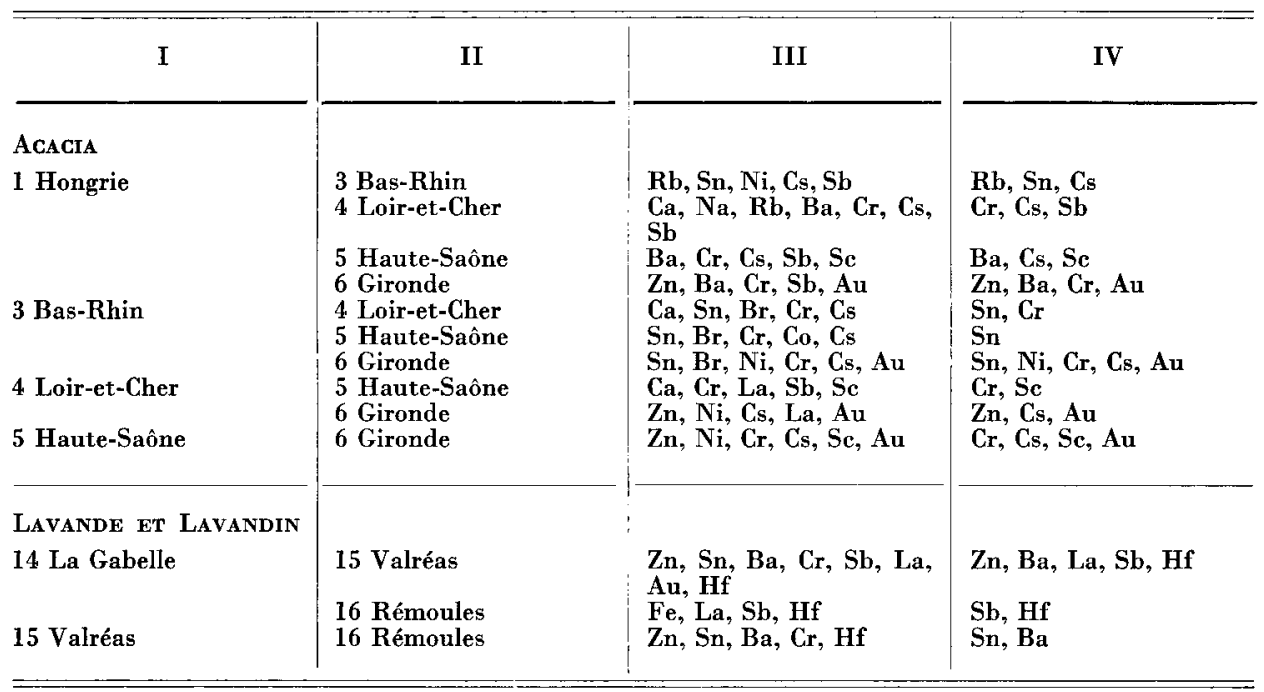

\begin{tabular}{|c|c|c|c|}
\hline I & II & III & IV \\
\hline \multicolumn{4}{|l|}{ Callune } \\
\hline 11 Étang de Léon & 12 Région Sabres & $\begin{array}{c}\text { pas de différence } \\
\text { Kein Unterschied }\end{array}$ & $\begin{array}{l}\text { pas de différence } \\
\text { Kein Unterschied }\end{array}$ \\
\hline 12 Rég. de Sabres & $\begin{array}{l}13 \text { Cévennes } \\
13 \text { Cévennes }\end{array}$ & $\begin{array}{l}\mathrm{Cr}, \mathrm{La}, \mathrm{Sc} \\
\mathrm{Zn}, \mathrm{La}, \mathrm{Sc}\end{array}$ & $\begin{array}{l}\mathrm{La}, \mathrm{Sc} \\
\mathrm{Zn}, \mathrm{La}, \mathrm{Sc}\end{array}$ \\
\hline \multicolumn{4}{|l|}{ Sapin } \\
\hline $\begin{array}{l}7 \text { Loire } \\
8 \text { V. de Munster }\end{array}$ & $\begin{array}{l}8 \text { V. de Munster } \\
9 \text { Raon l'Étape } \\
10 \text { Ballon d'Alsace } \\
9 \text { Raon l'Étape }\end{array}$ & $\begin{array}{l}\mathrm{Sn}, \mathrm{Ag} \\
\mathrm{Zn}, \mathrm{Rb}, \mathrm{Cs}, \mathrm{Au}, \mathrm{Ag} \\
\mathrm{Ca}, \mathrm{Zn}, \mathrm{Sb}, \mathrm{Au}, \mathrm{Ag} \\
\mathrm{Zn}, \mathrm{Sn}, \mathrm{Cs}, \mathrm{Co}\end{array}$ & $\begin{array}{l}\mathrm{Ag} \\
\mathrm{Cs}, \mathrm{Au}, \mathrm{Ag} \\
\mathrm{Ca}, \mathrm{Zn}, \mathrm{Au}, \mathrm{Ag} \\
\text { pas de différence } \\
\text { Kein Unterschied }\end{array}$ \\
\hline 9 Raon l'Étape & $\begin{array}{l}10 \text { Ballon d'Alsace } \\
10 \text { Ballon d'Alsace }\end{array}$ & $\underset{\mathrm{Cs}}{\mathrm{Zn}, \mathrm{Sn}, \mathrm{Au}}$ & $\begin{array}{l}\mathrm{Zn} \\
\mathrm{Cs}\end{array}$ \\
\hline
\end{tabular}


toutes les concentrations sont égales à $50 \%$ près (facteur 3 ) et à plus forte raison à $66,6 \%$ près (facteur 5 ). Il n'y a pas de distinction possible. Par contre la teneur en calcium du miel no $4(3,2$ ppm) multipliée par 1,5 pour tenir compte du taux de variation de $50 \%$ supposé, reste inférieure à la plus petite concentration des miels $n^{\text {os }} 1,3$ et 5 , même divisée par 2 . La distinction est donc possible selon ce critère. Remarquons que, dans ces conditions, le calcium seul ne permet pas de séparer les miels nos 1,3 et 5 , ni le miel 6 d'avec les autres. Pour une marge portée à $66,6 \%$ il ne permet aucune séparation.

Les résultats sont repris dans le tableau 9 pour tous les miels d'une même catégorie pris deux à deux. Ainsi par exemple, le miel de Hongrie se distingue de celui du Bas-Rhin par cinq éléments : $\mathrm{Rb}, \mathrm{Sn}, \mathrm{Ni}, \mathrm{Cs}$, et $\mathrm{Sb}$ si l'on choisit un coefficient 3 , et seulement par $R b$, Sn et Cs avec un coefficient 5 .

Les deux séries de résultats obtenus avec les coefficients 3 et 5 sont tout à fait comparables, à une exception près : les miels de Sapin de la Vallée de Munster et de Raon l'Étape deviennent inséparables avec le facteur 5. Lorsque la valeur du rapport augmente, le nombre des éléments disponibles pour effectuer la séparation diminue ainsi que le pouvoir de discrimination de la méthode, à tel point que pour des valeurs suffisamment élevées seuls des miels de couleur différente pourront être distingués.

Ainsi avec un rapport de 9, soit $80 \%$ d'erreur relative, la répartition des miels de callune et de lavande reste inchangée. Le miel d'acacia du Bas-Rhin ressemble alors à celui de la Haute-Saône, tandis que les miels de Sapin d'Alsace et des Vosges deviennent identiques tout en restant bien différenciés de celui de la Loire grâce à l'argent.

Si le rapport devient égal à 20 , il est encore possible de distinguer les miels d'acacia et de lavande sans qu'il soit nécessaire d'avoir recours au mercure, seul élément de différence entre les miels du Bas-Rhin et de Valréas. Pour ce même rapport les deux miels foncés sont également séparés sauf ceux des Cévennes (callune), de la vallée de Munster et du Ballon d'Alsace (Sapin).

Enfin lorsque l'on choisit une valeur de 50 pour ce rapport, les seules distinctions possibles concernent la couleur; les miels clairs sont séparés des miels foncés avec une exception cependant : le miel du Bas-Rhin (acacia) ressemble aux miels de Raon l'Étape (Sapin) et des Cévennes (Callune).

\section{CONCLUSION}

L'analyse par radioactivation aux neutrons thermiques permet sans séparation chimique, la reconnaissance d'une vingtaine d'éléments traces dont les concentrations sont le plus souvent inférieures au ppm. Si le contenu minéral d'un miel, tributaire de celui du sol sur lequel fleurit une plante et de la plante 
elle-même qui joue le rôle d'un filtre plus ou moins sélectif, reste compris entre deux limites indépendantes de la date de la récolte, il doit permettre d'obtenir des renseignements sur la nature et l'origine géographique de l'échantillon étudié. Il serait donc d'un grand intérêt de pouvoir poursuivre les recherches pendant plusieurs années sur des échantillons ayant la même origine géographique mais récoltés à divers moments de l'année.

Reçu pour publication en avril 1974.

Eingegangen im April 1974.

\section{ZUSAMMENFASSUNG}

Gegenstand der vorliegenden Arbeit ist die Untersuchung etwaiger engerer Beziehungen zwischen den mineralischen Bestandteilen eines Honigs und seinem geographischen Ursprung. Der geringe Gehalt an Mineralspuren in solchen Untersuchungsproben erfordert eine ganz besondere Analysentechnik, die Radioaktivierung, die sich vor allem, bei Ausschaltung chemischer Vorgänge, durch eine sehr grosse Empfindlichkeit auszeichnet.

- Die Aschen von je 15g Honig werden während 72 Stunden im thermischen Neutronenstrom der Atombatterie « Pegasus » im Centre d'Etudes Nucléaire von Cadarache bestrahlt. Danach werden mehrere Spektralanalysen ausgeführt, deren Ergebnisse im Computer vararbeitet werden, um verschiedene Gammalinien festzustellen und ihre Eigenschaften zu bestimmen.

In den verschiedenen, von uns analysierten Honigen haben wir etwa 20 Spurenelemente festgestellt, meist in einer Konzentration geringer als p.p.m. Einige der isolierten Elemente können “ interessante Anzeiger » sein, z.B. Quecksilber in den Lavendelhonigen oder Silber im Tannenhonigtauhonig des Loiregebietes. Die Beziehungen $R b / F e$ oder $R b / Z n$ sind charakteristisch für gewisse Honigsorten. Sie ermöglichen die Unterscheidung von hellen und dunklen Honigen oder auch des ungarischen vom französischen Akazienhonig. Schlieslich lassen sich Honige auf grund der relativen Bedeutung eines oder mehrerer ihrer Spurenelemente von einander unterscheiden.

- Es handelt sich hier um eine vorläufige Arbeit, die noch genauer durchgeführt und vertieft werden muss. Auf alle Fälle aber kann der Schluss gezogen werden, dass die mineralische Zusammensetzung eines Honigs vertvolle Hinweise auf seine Natur und seine geographische Herkunft ermöglicht.

\section{RÉFÉRENCES BIBLIOGRAPHIQUES}

Bryan D. E., et al., 1967. - Nuclear Activation Techniques in the life Sciences, Proc. Symp. Amsterdam. A.I.E.A. Vienne, 1967, 681-694.

Fehlmann C., 1912. - Beitrag zur Kenntnis der mineralischen Bestandteile des Honigs. Schweiz. Bienenztg. 48, 129-136.

Junod E., 1970. - Tables de constantes des Réactions et Isotopes. Communication personnelle. Koch R. C., 1960. - Activation analysis handbook. Acad. Press, New York.

Louveaux J., 1968. - Traité de biologie de l'abeille. Vol. 3., 325-362. Masson Éd. Paris.

Louveaux J., Maurizio A., Vorwohr G., 1970. — Les méthodes de la mélisso-palynologie. Ann. Abeille 1 (2), 211-227.

Misкiewicz W., 1969. - Main mineral constituants of polish honeys. Chem. abstr., 71, 29-390 d. 
MLadenov S., 1968. - Éléments majeurs et oligoéléments du miel et leurs effets sur l'homme (en serbo-croate) - Hrana ishhrana, 9, 461-67. (Ext. Chem. Abstr., 1969, 71, 2250 w.).

Pannisser J. C., 1973. - Code de traitements sur ordinateur des Histogrammes d'amplitude obtenus avec Semicteurs Ge-Li. Programme Jonque Note CEA N 1578.

Pappas A. C., Alstad J., Gulbrand L., 1963. - Détermination of traces elements in Opium by means of activation analysis. Radiochimica acta I (3), 109-117.

Perrov V., 1970. - Mineral constituants of some Australian honeys as determined by atomic absorption spectrophotometry. J. Apicult. Res., 9 (2), 95-101.

Pourtallier J., 1967. - Détermination quantitative des sucres des miels par chromatographie en phase gazeuse. Bull. apicole $10(2), 209-212$.

Shuette H. A., RÉmY K., 1932. - Degree of pigmentation and its probable relationship to the mineral constituants of honeys. J. Am. Chem. Soc., 54, 2909-2913.

Shuette H. A., Triller R., 1938. - Mineral constituants of honey. III Sulphur and chlorine. Fd. Res, 3 (5), 543-547.

Shuette H. A., Woessner W. W., 1939. - Mineral constituants of honey. IV Sodium and Potassium. Fd. Res. 4 (44), 349-353.

Van Dine D. L., Thompson A. R., 1908. - Hawaiian honeys. Bull. Hawaii agric. exp. Stn. No. 17.

VARJU M., 1970. - Teneur en éléments minéraux du miel d'acacia hongrois et corrélation avec la plante et le sol. Z. Lebensm. Unters. Forsh. 144 (5) 308-312.

Vermeulen L., Pelenents C., 1965. - Teneur en sucres, en phosphore et en fer de miels d'origine belge. Medel, landbouwhogesch, Gent. 30 (2), 527-541. 